\section{Kansans in the Middle of the Pandemic: Risk Perception, Knowledge, Compliance with Preventive Measures, and Primary Sources of Information about COVID-19}

\author{
Mugur V. Geana, M.D., Ph.D.
}

University of Kansas, Lawrence, KS

William Allen White School of Journalism and Mass Communication

Received May 28, 2020; Accepted for publication. June 15, 2020; Published online June 25, 2020

\section{ABSTRACT}

Introduction. As we conduct this study, the world is in the grasp of a deadly pandemic. In less than six months since its first diagnosis in Wuhan, China, the COVID-19 infectious disease due to the novel coronavirus has infected over 5,000,000 people and claimed over 350,000 lives. In the United States, most of the cases are in large urban settings along the coasts, but the disease is slowly progressing through the mainland. Kansas, with its particular location in the midwest United States, has seen a relatively small number of cases, but these are increasing. The Kansas government took radical measures to prevent the spread of the disease. According to the Health Beliefs Model, an individual's perception of risk will dictate engagement with preventive behaviors. Knowledge about the disease and preventive measures drive the risk assessment. Knowledge is dependant on the sources of information used. This study explored these metrics in a sample of Kansans living in the times of the COVID-19 pandemic.

Methods. A combination of snowball samples and random distribution through social media was used to recruit participants to an online survey. The risk and knowledge instrument was developed and validated by WHO Europe. Data collection lasted 96 hours.

Results. The attitudes and behaviors of Kansans concerning COVID19 were consistent with its location in an area of the country with a relatively lower incidence of the disease. Participants had good knowledge about the disease and preventive measures and were willing to comply with recommendations from local authorities.

Conclusion. Localized information sources that cater to the community are often primary, while social media is not a valuable source for information pertinent to COVID-19. Kans J Med 2020;13:160-164

\section{INTRODUCTION}

The COVID-19 pandemic, first documented in Wuhan, China, in December 2019, has left a significant mark on the world. ${ }^{1}$ As of May 2020, almost four million confirmed cases have been diagnosed worldwide, and 270,000 people have died due to the disease. These numbers keep rising. The pandemic had a fundamental impact on all layers of society, with significant disruptions to national and international travel, market indexes, industrial and service activities, education, and employment. ${ }^{2-4}$ Currently, there is no effective treatment and no vaccine. ${ }^{5}$ Although over 1.2 million people diagnosed with the coronavirus infection have recovered, the World Health Organization (WHO) has suggested that these patients may lack long-term immunity to the disease.

China seems to have done the best job of containing the local epidemic by implementing strict social distancing rules, combined

\section{KANSAS JOURNAL of MEDICINE}

with testing and aggressive contact identification and tracking activities. ${ }^{6,7}$ These measures were successful in maintaining the number of recorded cases to under 100,000 , with a mortality rate of about $2 \%$. Unfortunately, the acceleration phase of the local Chinese epidemic coincided with the celebration of the Chinese New Year, thus impacting travelers from all over the world. Subsequent infection foci have appeared in Japan, Iran, Europe, and the United States. Europe was considered the epicenter of the pandemic for the first three weeks in March, with Italy and Spain being the hardest-hit countries. The United States was ranked first on March 26, 2020, and it was considered the world's region with the most vigorous coronavirus activity. ${ }^{8}$

With a global impact on human society on multiple levels, estimating knowledge about the pandemic, protective measures, and what sources people are using to get information about it becomes of heightened importance. This study aims to explore these metrics in a sample of Kansans living in the times of the COVID-19 pandemic. According to the U.S. Centers for Disease Control and Prevention (CDC), on May 7,2020, the United States officially recorded 1,219,066 confirmed cases and 73,297 fatalities. ${ }^{9}$

Twenty-two U.S. states reported more than 10,000 cases of COVID-19.10 With 6,501 established COVID-19 patients, the state of Kansas is ranked 31st for the number of infections. Because of its demographics, Kansas has a particular bi-polar geographical distribution of patients with coronavirus infection, with three counties in the soutwestern part of the state (Finney, Seward, and Ford counties) accounting for approximately $36.5 \%$ of all cases. Four counties in eastern Kansas (Lyon, Shawnee, Wyandotte, and Johnson) accounted for about $30 \%$ of all cases. With a few exceptions, most of the 105 Kansas counties have recorded less than ten confirmed cases of COVID-19 each. The number of fatalities for the entire state, as of May 8, 2020, stood at 177.

Kansas, as well as most U.S. states and most countries around the world, issued stay-at-home orders to promote social distancing to stop the accelerated spread of the disease." The purpose was to avoid overwhelming healthcare services with an abrupt increase in the number of COVID-19 patients that would require hospitalization and intensive care. The closure of all educational facilities, manufacturing, and service businesses further enhanced these orders, with only essential workers being allowed to go to work. Many companies transitioned all their operations to employees working from home, and restaurants either closed or only offered take-out service. The essential businesses that were open (healthcare, pharmacies, sanitation, grocery stores, and other food services) imposed strict social distancing rules following the state and CDC guidelines. Kansas counties canceled all social gatherings and events.

As a result, the number of coronavirus infections seemed to be under relative control, Kansas being in the lower half of U.S. states for the number of confirmed COVID-19 cases and deaths. ${ }^{12}$ Although not enforced, stay at home orders seem to have been adopted by 
KANSAS JOURNAL of MEDICINE

KANSAS IN THE MIDDLE OFTHE PANDEMIC continued.

most Kansas residents. These social challenges imposed by the COVID-19 have had profound implications on the daily life of many Kansans..$^{13}$ Imposed or voluntary social distancing may have altered how Kansans gather news, their perception of information sources, which in exchange may have influenced their knowledge about, and perception of risk toward the pandemic. Gauging population beliefs, attitudes, and behaviors related to the COVID-19 pandemic is paramount for adequate public health engagement and contingency planning. The World Health Organization recommended periodic assessments, especially considering the fluid situation associated with these infectious events and the large variability of social, economic, political, and environmental factors that play a role in fighting the COVID-19 pandemic.

Data for this study comes from respondents from Kansas that took part in a national survey conducted by the authors. The current study aimed to answer the following research questions:

RQ1: What is the perception of risk related to COVID-19?

RQ2: What is the level of knowledge about COVID-19?

RQ3: To what degree do Kansans accept recommended behavior to protect from infection?

RQ4: What are the primary sources of information about COVID-19?

RQ5: Are there any differences in risk perception, knowledge, and use of primary sources of information based on gender, education level, place of work, or habitat (urban/rural)?

\section{METHODS}

This research project was approved by the Institutional Review Board of the University of Kansas.

A dedicated questionnaire distributed through an online survey collected data for the study. The researchers employed a combination of snowball sampling and direct promotion via social media (Facebook) to reach respondents all over the U.S. and create a convenience sample. The authors minimally adapted the risk perception questionnaire from an instrument developed and validated by WHO Europe to measure behavioral insights about COVID-19. ${ }^{14}$ WHO Europe developed the primary tool in collaboration with the University of Erfurt, Germany, and the research consortium COSMO group. The adaptation of the questionnaire used in this study was limited, to ensure the language and references were adequate for U.S. audiences (for example, "novel coronavirus" was changed to "COVID-19", administrative divisions were adjusted accordingly, etc.). The media sources classification used in the study reflected the current reality in information mediums in the U.S., and it was similar to that used on several other studies on health information media usage and trust. ${ }^{15-16}$ As the epidemic was approaching its apex in the U.S., and information changed almost daily, data collection was limited to a period of 96 hours.

The national sample was of 542 respondents. Of these, 131 participants were from Kansas. The following analysis focused solely on
Kansas residents.

The mean age for the sample was 46 years ( $+/-15.5$ years), and $81.7 \%$ were female respondents. Participants mostly were highly educated, with $86 \%$ of participants having completed a college degree. Seventeen percent of the sample were college students. Of the 131 respondents, $59 \%$ lived in urban or suburban areas, and $41 \%$ lived in rural areas. Twenty-seven percent were working in a medical facility or other places where they would come in direct contact with COVID-19 patients. Forty percent of those surveyed lived in towns of less than 20,000 inhabitants, and $86 \%$ reported themselves as whites. Twenty-seven percent stated an annual income of less than $\$ 50,000$, $29 \%$ declared an income between $\$ 50,001$ and $\$ 100,000$, and $44 \%$ had incomes over $\$ 100,000$.

\section{RESULTS}

None of the people taking the survey have had a coronavirus infection; twenty stated that they did not know if they had it. Although only $8 \%$ said that they had family members confirmed as having COVID$19,41 \%$ of the sample knew people in their community or place of work sick with COVID-19. Fifteen percent were aware of fatalities in their community or place of work because of the epidemic. A risk perception index, constructed out of four questions addressing risk (scale 4 - minimum to 40 - maximum), showed a normal distribution for the Kansas sample of participants. A Kolmogorov-Smirnov test indicated that the risk perception index followed a normal distribution, $\mathrm{D}(123)=0.08, \mathrm{p}=0.066$. Most respondents assessed their risk of getting infected with the novel coronavirus as average.

Kansans reported their knowledge of COVID-19 as above average, most stating that they had good or excellent knowledge about these topics $(\mathrm{D}(129)=0.146, \mathrm{p}<0.001$ for COVID-19 knowledge, and $\mathrm{D}(129)=0.206, \mathrm{p}<0.001$ for knowledge about preventive measures). These observed results stood even when controlling for participants that declared they work in a medical facility dealing daily with COVID-19 patients, thus assuming a higher level of knowledge on both variables. To further the analysis, two additive COVID-19 knowledge indexes were computed for basic knowledge and knowledge about transmission pathways. Both indexes showed that Kansan participants in the survey have knowledge scores toward the higher end of the knowledge spectrum, even when controlling for those working in medical facilities. There is a positive statistically significant correlation between perceived knowledge and measured basic knowledge $(\mathrm{r}(95)=0.283, \mathrm{p}=0.006)$, but not between perceived knowledge and knowledge about transmission pathways for the virus.

For Kansans working in the medical field, there was a significant positive statistical correlation between their self-assessed knowledge and both the basic and transmission knowledge indexes (r(34) $=0.422, \mathrm{p}=0.013$, and $\mathrm{r}(34)=0.532, \mathrm{p}=0.001$, respectively). Nevertheless, an ANOVA test showed that the measured knowledge differences between those working in the medical field, and the other respondents, were not statistically significant. When it came to following authorities' recommendations to prevent the spread of COVID-19, most Kansans in our sample stated that they follow them; $80 \%$ of participants placed in the upper quartile of a 1 to 10 scale measuring compliance with preventive measures $(D(130)=0.244$, 
measuring compliance with preventive measures $(\mathrm{D}(130)=0.244$, $\mathrm{p}<0.001$ ). Answers to questions about preventive measures against COVID-19 showed that over $97 \%$ of our sample stated that they wash their hands for over 20 seconds, and most of them do not find that a major inconvenience. Over $90 \%$ of those surveyed said that they avoid touching their face, and use disinfectants to clean their hands when water and soap are not available. Fifty-four percent stated that they use caution when manipulating and opening mail. When asked specifically about the use of a face mask when going out in public, only $37 \%$ of respondents stated that they always use a face mask, while $41 \%$ said they would use a face mask sometimes, and $23 \%$ had never used one. There was a statistically significant correlation between mask usage when going out in public and the perception of the usefulness of a mask to prevent the spread of COVID-19. Those declining to use a mask seemed to have a stronger opinion about masks not being useful $(\mathrm{r}(128)=-0.32, \mathrm{p}<0.001)$. Kansans in our sample were worried about both their safety as well as that of others: $74 \%$ strongly agreed that they use social distancing to protect themselves, but $83 \%$ used it because they want to protect others.

In times of need, people desired to know what was happening in their community and the world. Previous research has shown that often multiple sources are employed to gather information about a certain topic; the survey asked about the usage of sources identified as providing information about COVID-19. ${ }^{17}$ The daily COVID-19 update by Kansas Governor Laura Kelly was the primary source of information for those answering our survey, with almost $80 \%$ of participants following it almost daily. News from search engines such as Google or Bing ranked second, with $60 \%$ of respondents using them daily. News from national newspapers (e.g., New York Times, Wall Street Journal) ranked third, being used on an almost daily basis by over half of participants. Family, friends, and coworkers were important sources of information as well as an opportunity to discuss news and events. Over $50 \%$ of our sample had these daily conversations. Consultations with health care workers for information about COVID-19 happened almost daily for $36 \%$ of those in our sample who were not working in a healthcare facility. For those working in a medical environment, conversations with colleagues about COVID19 ranked as their first source of information, even surpassing the

\section{KANSAS: Trust in Sources of Information about COVID-19}

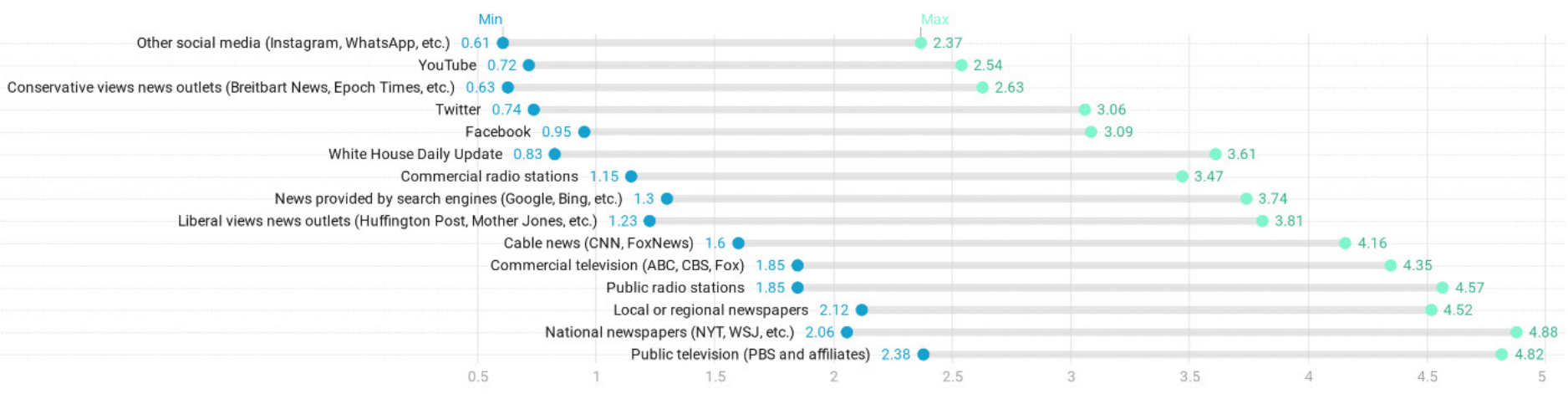

Figure 1. Trust in mediated sources of information about COVID-19 among a sample of Kansas residents.
KANSAS JOURNAL of MEDICINE

KANSAS INTHE MIDDLE OFTHE PANDEMIC

continued.

daily report by the Governor. Surprisingly, the least used source of information about COVID-19 by Kansans was the White House's daily update on the outbreak. Participants were asked to rank their trust in information sources on a five-star ranking system, with one star being "No Trust At All" and five stars being "A Great Deal Of Trust" (Figure 1). Public television and national newspapers were the most trusted media sources of information about COVID-19, and social media sources were the least trusted.

Only one respondent in our dataset did not identify with a specific gender. Gender comparison was conducted only on those who identified with a particular gender. As mentioned earlier, 23 participants identified as male and 107 as female. An ANOVA test did not show any statistically significant differences between genders regarding risk perception, basic, or transmission knowledge about COVID-19. Independent samples t-test showed that while females were more prone to use a mask when going out in public, the differences were not statistically significant. There were small, but statistically significant differences $(\mathrm{t}(127.8)=4.098, \mathrm{p}<0.001)$ in the perception of the value of social distancing to protect one's self between men $(\mathrm{M}=5.96, \mathrm{SD}=$ $0.209)$ and women $(\mathrm{M}=5.54, \mathrm{SD}=0.945)$. Similar findings held for the benefit of social distancing to protect others $(\mathrm{t}(127.5)=2.867, \mathrm{p}=$ 0.005). Gender did not generate significant differences in the usage of various sources for retrieving information about COVID-19 or for the trust in those sources. Although the education level was an independent variable that we expected to analyze, the skewed sample towards highly educated participants did not allow us to perform this analysis. Respondents working in a medical facility $(\mathrm{M}=23.47, \mathrm{SD}=$ 5.86) have a higher perception for risk of getting COVID-19 ( $\mathrm{t}(120)$ $=3.287, \mathrm{p}=0.001)$ than those who do not work in a healthcare unit $(\mathrm{M}=19.27, \mathrm{SD}=6.13)$.

At first glance, participants living in rural areas of the state $(\mathrm{M}=22$, $\mathrm{SD}=6.28$ ) had a higher perception of risk associated with COVID-19 $(\mathrm{t}(128)=-2.4, \mathrm{p}=0.018)$ than those living in urban areas $(\mathrm{M}=19.25$, $\mathrm{SD}=6.12$ ). When controlling for the place of work (medical facility), there were no statistically significant differences between those living 
KANSAS JOURNAL of MEDICINE KANSAS IN THE MIDDLE OFTHE PANDEMIC continued.

in urban areas $(\mathrm{M}=19.25, \mathrm{SD}=6.12)$. When controlling for the place of work (medical facility), there were no statistically significant differences between those living in rural or urban areas concerning the perception of risk. Participants from rural areas seemed to use conservative views news outlets, Facebook, YouTube, and commercial radio stations more than those living in urban areas. As a source of information, those living in rural areas also seemed to trust more the White House Daily Update on COVID-19, YouTube, commercial radio stations, and conservative views news outlets than those living in urban areas.

\section{DISCUSSION}

Our first research question asked about the risk perception related to COVID-19. The results showed that most Kansans in our sample perceived their overall risk of getting COVID-19 disease as average. At the time of the survey, Kansas ranked in the lowest third of all U.S. states as the number of confirmed infections, and a stay at home measure was in full force. After controlling for healthcare workers, many participants estimated that avoiding disease would be easy for them. These findings were consistent with previous research that showed people's risk perception related to an infectious disease was dependent, among other things, on the severity of the outbreak in their communities. ${ }^{18}$

Our second research question explored knowledge about COVID19. The survey looked at both self-assessed knowledge level as well as actual knowledge about the main characteristics of the COVID19 outbreak (e.g., infectiousness, transmission). Participants had an above overall average self-assessment of their understanding of COVID-19, which also was consistent with the findings of the instrument measuring basic COVID-19 knowledge. The salience of the topic in the news and the local rules governments imposed in response to the outbreak partially may explain these findings. ${ }^{19}$ Kansans in our sample who worked in medical facilities had a better understanding of preventive measures.

The third research question looked at Kansans' acceptance of recommended protective behavior against COVID-19. Our findings showed a high level of compliance with preventive practices. Previous research has demonstrated that a relationship exists between knowledge and preventive action, ${ }^{20}$ especially for higher educated audiences. ${ }^{21-22}$ Similar to previous research looking at beliefs about the efficacy of a preventive behavior and embracing said practice, ${ }^{23}$ those participants in our sample who do not believe in the value of wearing a mask were less likely to use one when going out in public.

The fourth research question explored the use of primary information sources. Kansas is known as a "sentinel" state, with its people focused on hard work, traditional values, and a focus on their community. ${ }^{24}$ Although most participants used multiple sources, the daily updates on the COVID-19 pandemic provided by the local administration were the primary information source used. Search engines, due to their convenience and ease of use, ranked second, followed by consecrated sources such as national newspapers. Social media was the least information source that Kansans would turn to for information about COVID-19. Recent scandals that have plagued social media and issues with privacy and misinformation may explain these findings. ${ }^{25-26}$ Social media sources also were among the least trusted sources of information for survey participants. Similar to findings in other studies, families and friends, coworkers, and medical professionals were a significant source of information about COVID-19, with health care workers being the most trustworthy source. ${ }^{27}$

The last research question focused on the impact of some demographic variables on risk perception and knowledge about COVID-19. The results showed minor effects of gender on risk perception and the value of social distancing, with men seemingly being more compliant than women. There was no influence of gender on the utilization or trust of information sources. Working in a medical facility in Kansas and being daily at risk of exposure to the novel coronavirus significantly increased risk perception. Respondents living in rural areas tended to use more conservative media outlets than those in urban developments.

Limitations, Conclusion, and Future Research. This study was not without limitations. The use of a convenience sample was probably the main impediment to the generalizability of results. Facebook has become an essential tool for research in social sciences. ${ }^{28}$ Although the survey only reached those having Facebook accounts, by combining snowball sampling with the random display of the link to the study through Facebook advertising, the reach was expanded to the 1,800,000 Facebook accounts in Kansas. Facebook is a viable platform for participant recruitment and data collection, and results can be generalized as differences to random samples from the same population are nonsignificant in magnitude..$^{29}$ In some situations, snowball sampling using Facebook provided better results than the traditional snowball sampling method. ${ }^{30}$ Even as we would have liked a larger sample, we only collected data over 96 hours, and its size was adequate to perform all statistical calculations for this study. Like with everything else, willingness to participate in research resides with the potential participant, equally valid for any type of data collection method. We had a highly educated sample, which is somewhat consistent with similar results for social media studies that do not offer participant compensation. ${ }^{31}$ The same can be said about the gender of respondents, females being more willing to participate in online surveys than males. ${ }^{32}$

The attitudes and behaviors of Kansans concerning COVID-19 were consistent with its location in an area of the country with a relatively lower incidence of the disease. Participants in our survey had good knowledge about the disease and preventive measures and were willing to comply with recommendations from local authorities. Localized information sources that cater to the community were often primary, while social media was not a valuable source for information pertinent to COVID-19. Kansas was still in the acceleration phase of the epidemic as this study was conducted. Future research should look at how long time mandatory isolation may influence attitudes and behaviors about COVID-19. As families feel the economic impact of social distancing, research should look if people migrate towards more partisan media sources. Consistent with the 
recommendations of the WHO team that developed the instrument used in this research, preliminary information was shared about our findings with the public health authorities of Johnson County and the Kansas City Unified Government within two days from the completion of the survey.

\section{REFERENCES}

${ }^{1}$ Chen N, Zhou M, Dong X, et al. Epidemiological and clinical characteristics of 99 cases of 2019 novel coronavirus pneumonia in Wuhan, China: A descriptive study. Lancet 2020; 395(10223):507-513. PMID: 32007143.

${ }^{2}$ Baker SR, Bloom N, Davis SJ, Kost KJ, Sammon MC, Viratyosin T. The unprecedented stock market impact of COVID-19. March 27,2020. https:// www.policyuncertainty.com/media/StockMarkets_COVID.pdf.

${ }^{3}$ Nicola M, Alsafi Z, Sohrabi C, et al. The socio-economic implications of the coronavirus pandemic (COVID-19): A review. Int J Surg 2020; 78:185-193. PMID: 32205533.

4 Zhang Y, Ma ZF. Impact of the COVID-19 pandemic on mental health and quality of life among local residents in Liaoning Province, China: A crosssectional study. Int J Environ Res Public Health 2020; 17(7):2381. PMID: 32244498.

${ }^{5}$ Ahn D-G, Shin H-J, Kim M-H, et al. Current status of epidemiology, diagnosis, therapeutics, and vaccines for novel coronavirus disease 2019 (COVID-19). J Microbiol Biotechnol 2020; 30(3):313-324. PMID: 32238757.

${ }^{6}$ Chinazzi M, Davis JT, Ajelli M, et al. The effect of travel restrictions on the spread of the 2019 novel coronavirus (COVID-19) outbreak. Science 2020; 368(6489):395-400. PMID: 32144116.

7 Yang CY, Wang J. A mathematical model for the novel coronavirus epidemic in Wuhan, China. Math Biosci Eng 2020; 17(3):2708-2724. PMID: 32233562.

8 CBS News. Coronavirus Updates from March 26, 2020. CBS News, May 28. https://www.cbsnews.com/live-updates/coronavirus-news-latest-2020-03-26/.

9 US Centers for Disease Control and Prevention. Coronavirus Disease 2019 (COVID-19). May 8, 2020. https://www.cdc.gov/coronavirus/2019-ncov/ cases-updates/cases-in-us.html.

${ }^{10}$ Bing.com.COVID-19 Tracker. 2020.https://www.bing.com/covid/local/ kansas_unitedstates.

${ }^{11}$ Kansas Office of the Governor. Governor Kelly issues temporary, statewide Stay Home order in ongoing effort to combat COVID-19.2020. https:/ governor.kansas.gov/governor-kelly-issues-temporary-statewide-stayhome-order-in-ongoing-effort-to-combat-covid-19/. Accessed May 28, 2020 .

${ }^{12}$ Microsoft. COVID-19 Tracker.2020.https://www.bing.com/covid/local/ unitedstates. Accessed May 26, 2020.

13 ZieglerL.InKansas City, Kansas, PeopleBeginAdjusting ToLifeAmidCoronavirus. March 2l, 2020. https://www.kcur.org/community/2020-03-2l/ in-kansas-city-kansas-people-begin-adjusting-to-life-amid-coronavirus. Accessed June 9, 2020.

${ }^{14}$ World Health Organization Europe. WHO tool for behavioural insights on COVID-19. April 17, 2020. http://www.euro.who.int/en/health-topics/ health-emergencies/coronavirus-covid-19/novel-coronavirus-2019-ncovtechnical-guidance-OLD/who-tool-for-behavioural-insights-on-covid-19.

15 Smith D. Health care consumer's use and trust of health information sources. J Commun Healthc 2011; 4(3):200-210.

${ }^{16}$ Catalán-Matamoros D, Peñafiel-Saiz C. The use of traditional media for public communication about medicines: A systematic review of characteristics and outcomes. Health Commun 2019; 34(4):415-423. PMID: 29252009. ${ }_{17}$ Sbaffi L, Zhao C. Modeling the online health information seeking process: Information channel selection among university students. J Assoc Inf Sci Tech 2020; 71(2):196-207.

${ }^{18}$ Ibuka Y, Chapman GB, Meyers LA, Li M, Galvani AP. The dynamics of risk perceptions and precautionary behavior in response to $2009(\mathrm{HINl})$ pandemic influenza. BMC Infect Dis 2010; 10(1):296. PMID: 20946662.

${ }_{19}$ Ho SS, PehX, Soh VW. The cognitive mediation model: Factors influencing public knowledge of the $\mathrm{HlNl}$ pandemic and intention to take precautionary behaviors. J Health Commun 2013; 18(7):773-794. PMID: 23402299.

${ }^{20}$ Gomes APM, da Silva EG, Gonçalves SHF, et al. Relationship between patient's education level and knowledge on oral health preventive measures. Int Dent Med J Adv Res 2015; 1(1): 1-7.

${ }^{21}$ Taghrir MH, Borazjani R, Shiraly R. COVID-19 and Iranian medical students; A survey on their related-knowledge, preventive behaviors and risk perception. Arch Iran Med 2020; 23(4):249-254. PMID: 32271598.
KANSAS JOURNAL of MEDICINE

KANSAS IN THE MIDDLE OFTHE PANDEMIC continued.

22 Van Benthem BHB, Khantikul N, Panart K, Kessels PJ, Somboon P, Oskam L. Knowledge and use of prevention measures related to dengue in northern Thailand. Trop Med Int Health 2002; 7(11):993-1000. PMID: 12390606.

${ }^{23}$ Cherrington A, Corbie-Smith G, Pathman DE. Do adults who believe in periodic health examinations receive more clinical preventive services? Prev Med 2007; 45(4):282-289. PMID: 17692368.

${ }^{24}$ 16Personalities.com. Personality Geography of the United States. https:// www.l6personalities.com/articles/personality-geography-of-the-unitedstates.

${ }^{25}$ Jozani M, Ayaburi E, Ko M, Choo K-KR. Privacy concerns and benefits of engagement with social media-enabled apps: A privacy calculus perspective. Comput Human Behav 2020; 107:106260.

${ }^{26}$ Mian A, Khan S. Coronavirus: The spread of misinformation. BMC Med 2020; 18(1):89. PMID: 32188445.

27 Geana MV, Kimminau KS, Greiner KA. Sources of health information in a multiethnic, underserved, urban community: Does ethnicity matter? J Health Commun 2011; 16(6):583-594. PMID: 21391043.

${ }^{28}$ Kosinski M, Matz SC, Gosling SD, Popov V, Stillwell D. Facebook as a research tool for the social sciences: Opportunities, challenges, ethical considerations, and practical guidelines. Am Psychol 2015; 70(6):543-546. PMID: 2634336.

${ }^{29}$ Rife SC, Cate KL, Kosinski M, Stillwell D. Participant recruitment and data collection through Facebook: The role of personality factors. Int J Soc Res Methodol 2016; 19(1):69-83.

${ }^{30}$ Baltar F, Brunet I. Social research 2.0: Virtual snowball sampling method using Facebook. January 27, 2012. https://www.emerald.com/insight/ content/doi/10.1108/10662241211199960/full/html.

${ }^{31}$ Sheng X, Zolfagharian M. Consumer participation in online product recommendation services: Augmenting the technology acceptance model. J Serv Market 2014; 28(6):460-470.

${ }^{32}$ Smith WG. Does gender influence online survey participation?: A recordlinkage analysis of university faculty online survey response behavior. June 2008. https://eric.ed.gov/?id=ED501717.

Keywords: Kansas, COVID-19, pandemics, health knowledge, attitudes, practice, consumer health information 